\title{
Cytostatic versus cytocidal profiling of quinoline drug combinations via modified fixed-ratio isobologram analysis
}

\author{
Alexander P Gorka ${ }^{1,2}$, Lauren M Jacobs ${ }^{1}$ and Paul D Roepe ${ }^{1 *}$
}

\begin{abstract}
Background: Drug combination therapy is the frontline of malaria treatment. There is an ever-accelerating need for new, efficacious combination therapies active against drug resistant malaria. Proven drugs already in the treatment pipeline, such as the quinolines, are important components of current combination therapy and also present an attractive test bank for rapid development of new concepts.
\end{abstract}

Methods: The efficacy of several drug combinations versus chloroquine-sensitive and chloroquine-resistant strains was measured using both cytostatic and cytocidal potency assays.

Conclusions: These screens identify quinoline and non-quinoline pairs that exhibit synergy, additivity, or antagonism using the fixed-ratio isobologram method and find tafenoquine - methylene blue combination to be the most synergistic. Also, interestingly, for selected pairs, additivity, synergy, or antagonism defined by quantifying $I C_{50}$ (cytostatic potency) does not necessarily predict similar behaviour when potency is defined by $L D_{50}$ (cytocidal potency). These data further support an evolving new model for quinoline anti-malarials, wherein haem and haemozoin are the principle target for cytostatic activity, but may not be the only target relevant for cytocidal activity.

Keywords: Isobologram, Combination therapy, Quinoline, Cytocidal, P. falciparum

\section{Background}

Malaria remains a very serious threat to global health, with 300-500 million clinical cases and nearly 1 million deaths annually [1]. Given the growing spread of resistance to current anti-malarial drugs and lack of an effective vaccine [1-4], development of novel, cost-effective, and efficacious drugs is of utmost importance. Decades of use of drugs such as chloroquine $(\mathrm{CQ})$ have rendered several cost-effective quinoline monotherapies ineffective in various malaria endemic regions. For these reasons and others, the WHO currently recommends combination therapies, particularly artemisinin (ART)-based combination therapy (ACT), as first-line treatment for uncomplicated Plasmodium falciparum [5]. Currently valuable ACT consists of ART or derivatives, such as

\footnotetext{
* Correspondence: roepep@georgetown.edu

'Department of Chemistry, Department of Biochemistry, Cellular, \& Molecular Biology, and Center for Infectious Disease, Georgetown University, 37th and 'O' Sts. NW, Washington, DC 20057, USA

Full list of author information is available at the end of the article
}

dihydroartemisinin, artemether, and artesunate, in combination with longer half-life quinolines, such as amodiaquine $(\mathrm{AQ})$, mefloquine $(\mathrm{MQ})$, or quinine $(\mathrm{QN})$, antibiotics such as doxycycline and clindamycin, or the blood schizonticide lumefantrine $[2,4,5]$. Addition of a single dose of the 8 -aminoquinoline primaquine (PQ) to $\mathrm{ACT}$ is also recommended given its transmission blocking and gametocytocidal properties [5-7].

PQ was initially developed as a safer and more effective 8 -aminoquinoline alternative to plasmoquine, and is the only member of that drug class currently in use [6,7], although clinical trials with the related drug tafenoquine (TQ) are ongoing. PQ exhibits asexual stage activity against Plasmodium vivax [8], kills hypnozoites of P. vivax and Plasmodium ovale $[9,10]$, and is active against late-stage gametocytes of $P$. falciparum [6,7,11-13]. Studies have consistently shown that PQ can block transmission prior to the number of gametocytes being reduced [14-16]. PQ has poor growth inhibitory activity against intraerythrocytic stages of $P$. falciparum $[17,18]$, likely 
owing to poor inhibition of haemozoin $(\mathrm{Hz})$ formation in vitro [19-23] and an inability to bind ferriprotoporphyrin IX (FPIX) haem in solution [20].

Use of PQ is limited by short plasma half-life and toxicity, principally haemolysis in patients with $\mathrm{X}$-linked glucose-6-phosphate dehydrogenase (G6PD) deficiency [6,7], which varies between $3 \%$ and $30 \%$ in endemic areas [24]. Recent evidence has suggested that risk of PQ administration in high G6PD-deficient populations might be ameliorated by lower dosing, without loss of gametocytocidal potency $[25,26]$, which might be due to $P Q$ rapidly metabolizing to carboxyPQ, with plasma concentrations of the metabolite 10-fold greater than PQ $4 \mathrm{~h}$ post-dosing [27]. Tafenoquine (TQ) (WR238605), a 5phenoxyl derivative of PQ [28], has improved asexual stage activity [29], a significantly elongated plasma half-life (two weeks vs $6 \mathrm{~h}$ ) $[27,30]$, and a greater therapeutic index [31]. The compound remains haemolytic in G6PD-deficient individuals, though to a lesser extent than PQ [7].

In addition to use with current $\mathrm{ACT}$, coadministration of 8-aminoquinolines with blood schizonticides like CQ and QN have been explored for their anti-relapse and toxicity-reducing effects (see [32] for review). The latter may be due to competition between the 8 -aminoquinoline and the coadministered agent for cytochrome P450s, oxidases, or other metabolic enzymes [32]. Bray et al. reported that $\mathrm{PQ}$ and $\mathrm{TQ}$ act synergistically with $\mathrm{CQ}$ in vitro against the $\mathrm{CQ}$ resistant $(\mathrm{CQR}) \mathrm{K} 1$ strain, with no effect observed against the CQ sensitive (CQS) D10 strain [33], and that PQ analogues lacking the aminoalkyl side chain of PQ do not synergize with CQ. While this synergistic effect has not been quantified clinically or in vivo, there is some preliminary evidence that CQ combined with $\mathrm{PQ}$ can lead to decreased treatment failure rates against uncomplicated CQR P. falciparum [34,35]. PQ$\mathrm{CQ}$ synergy has also been proposed in treatment of CQR cases of $P$. vivax in Thailand $[8,36]$.

The thiazine dye methylene blue (MB) was the first synthetic compound ever used in the treatment of disease [37], and there has recently been a resurgence of interest in the anti-malarial properties of MB. The compound appears to have transmission-blocking effects via potent clearance of late-stage gametocytes [38], high blood schizonticidal activity [38-40], and $\mathrm{Hz}$ inhibition potential $[41,42]$, presumably due to accumulation in the digestive vacuole (DV) $[38,42]$ and/or redox-cycling potential $[41,43-45]$. MB has been observed to inhibit parasite glutathione reductase (GR) $[46,47]$, which could in theory enhance activity of drugs such as CQ that cause oxidative damage (see also [48]). However, Akoachere et al. demonstrated that $\mathrm{CQ}$ and $\mathrm{MB}$ act antagonistically against 3D7 (CQS) and K1 (CQR) strains [39]. Garavito et al. later reported this combination to be additive against strain FcM29 (CQR) [40]. Antagonism has also been reported in the field $[49,50]$, however, combination use with ARTs and AQ have proven effective [50,51]. Regardless, in in vitro studies, efficacy of these and other drug combinations has been via standard $P$. falciparum growth inhibition assays. No previous studies of drug combination effects have explicitly quantified potency in terms of cytocidal (parasite cell killing) activity.

Given the importance of combination therapy and the attractiveness of further development of combinations using proven, cost-effective, and widely-available drugs like $C Q, P Q$, and $M B$ (Figure 1), the effects of potentially interesting drug combinations on in vitro antiplasmodial cytostatic and cytocidal activity are investigated for both a CQS and CQR strain of $P$. falciparum. Use of isobologram-based approaches (see Methods) resolves conflicting accounts of synergy, additivity, and antagonism $[33,39,40]$, and probes the relationships between these parameters at cytostatic $v s$ cytocidal dosages [19,52]. The results suggest an important new concept relevant for the discovery of efficacious combination therapies.

\section{Methods}

\section{Materials and chemicals}

Routine chemicals, media, and solvents were reagent grade or better, purchased from Sigma-Aldrich (St. Louis, MO, USA) or Fisher Scientific (Newark, DE, USA), and used without further purification, unless otherwise noted. Sterile black flat bottom tissue culture, or non-sterile clear polystyrene 96-well plates, and all other laboratory plastics were from Fisher Scientific (Newark, DE). SYBR Green I nucleic acid stain was from Invitrogen (Eugene, OR, USA). Anti-malarial drugs AQ dihydrochloride dihydrate, CQ diphosphate, MB hydrate, PQ bisphosphate, and TQ succinate were from Sigma-Aldrich (St. Louis, MO, USA).

\section{Parasite culture}

Plasmodium falciparum clones HB3 (Honduras, CQS), Dd2 (Indochina, CQR), and FCB (Thailand/S. Africa) were obtained from the Malaria Research and Reference Reagent Resource Center (Manassass, VA, USA). Off-the-clot, heatinactivated pooled type $\mathrm{O}^{+}$human serum and type $\mathrm{O}^{+}$ human whole blood were purchased from Biochemed Services (Winchester, VA, USA). Custom $5 \% \mathrm{O}_{2} / 5 \% \quad \mathrm{CO}_{2} /$ $90 \% \mathrm{~N}_{2}$ gas blend was purchased from Robert's Oxygen (Rockville, MD, USA).

Plasmodium falciparum strains were maintained using the method of Trager and Jensen [53] with minor modifications. Briefly, cultures were maintained under an atmosphere containing $5 \% \mathrm{CO}_{2}, 5 \% \mathrm{O}_{2}$, and $90 \% \mathrm{~N}_{2}$ gaseous mix at $2 \%$ haematocrit and $1-2 \%$ parasitaemia in RPMI 1640 supplemented with $10 \%$ type $\mathrm{O}^{+}$human serum, $25 \mathrm{mM}$ HEPES (pH 7.4), $23 \mathrm{mM} \mathrm{NaHCO}$, 
<smiles>CCN(CC)CCCC(C)Nc1ccnc2cc(Cl)ccc12</smiles>

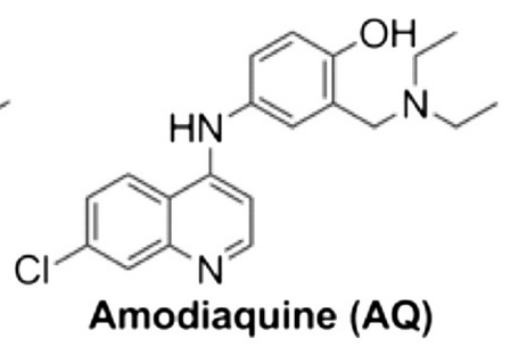<smiles>COc1cc(NC(C)CCCN)c2ncccc2c1</smiles><smiles>COc1cc(C)c2c(Oc3cccc(C(F)(F)F)c3)c(OC)cc(NC(C)CCCN)c2n1</smiles>

Tafenoquine (TQ)<smiles>CN(C)c1ccc2nc3ccc(=[N+](C)C)cc-3sc2c1</smiles>

Methylene Blue (MB)

Figure 1 Structures of anti-malarial drugs used in this study.

$11 \mathrm{mM}$ glucose, $0.75 \mathrm{mM}$ hypoxanthine, and $20 \mu \mathrm{g} / \mathrm{L}$ gentamicin, with regular media changes every $48 \mathrm{~h}$.

\section{Anti-plasmodial assays}

Anti-plasmodial cytostatic (growth inhibitory, quantified by the half-maximal growth inhibitory dose or $\mathrm{IC}_{50}$ ) and cytocidal (cell killing, quantified by the half-maximal lethal dose or $\mathrm{LD}_{50}$ ) activity was assessed against the above strains as previously described [52,54], with minor modifications. The cytocidal assay utilizes a $6 \mathrm{~h}$ bolus dose with high concentrations of drug followed by washing drug away and growth in the absence of drug for $48 \mathrm{~h}$, while the cytostatic assay utilizes continuous growth for $48 \mathrm{~h}$ in the constant presence of low concentrations of drug. Test compounds were dissolved in deionized water, $50 \%$ ethanol, or dimethyl sulfoxide. Serial drug dilutions were made using complete media and $100 \mu \mathrm{L}$ aliquots were transferred to 96-well clear-bottom black plates. Following addition of $100 \mu \mathrm{L}$ of asynchronous culture (1\% final parasitaemia, $2 \%$ final haematocrit), plates were transferred to an airtight chamber, gassed with $5 \% \mathrm{CO}_{2} / 5 \% \mathrm{O}_{2} / 90 \% \mathrm{~N}_{2}$ and incubated at $37^{\circ} \mathrm{C}$.

For the cytocidal assay, plates were incubated for $6 \mathrm{~h}$ followed by centrifugation with an Eppendorf 5804 centrifuge fitted with an A-2-DPW rotor (Hauppauge, $\mathrm{NY}$ ) at $700 \mathrm{~g}$ for $3 \mathrm{~min}$ [52]. Drug-containing media was removed and cell pellets washed three times with $200 \mu \mathrm{L}$ of complete media per wash, using the same centrifuge settings, and re-suspended in the same volume of media. Washed plates were incubated side by side with the cytostatic assay plates at $37^{\circ} \mathrm{C}$ for $48 \mathrm{~h}$. After $48 \mathrm{~h}, 50 \mu \mathrm{L}$ of 10X SYBR Green I dye (diluted using complete media from a 10,000X DMSO stock) was added and plates incubated for an additional $1 \mathrm{~h}$ at $37^{\circ} \mathrm{C}$ to allow DNA intercalation. Fluorescence was measured at $538 \mathrm{~nm}$ (485 nm excitation) using a Spectra GeminiEM plate reader (Molecular Devices; Sunnyvale, CA) fitted with a $530 \mathrm{~nm}$ long-pass filter. Linear standard curves of measured fluorescence $v s$ known parasitaemia were prepared immediately prior to plate analysis. Background controls included fluorescence from un-infected red blood cells. Data was analysed using MS Excel 2007 and $\mathrm{IC}_{50}$ and $\mathrm{LD}_{50}$ values obtained from sigmoidal curve fits to \% growth/survival vs drug concentration data using SigmaPlot 11.0. Reported values are the average of three independent assays, with each assay conducted in triplicate (nine determinations total) and reported \pm standard error of the mean (S.E.M.), unless otherwise noted. 


\section{Isobologram analysis}

The effect of drug combinations was assessed using the modified fixed-ratio isobologram analysis protocol [55] (hereafter referred to as "isobologram analysis"), with some modification. This method detects synergy, additivity, or antagonism between a drug pair. In general, if the drug pair has improved $\mathrm{IC}_{50}$ or $\mathrm{LD}_{50}$ potency relative to each drug alone, the combination is synergistic, if potency remains unchanged the effect is additive, and if potency is reduced the effect is antagonistic.

Master stock drug solutions were prepared for each drug using complete media, such that the final concentration approximates $\mathrm{IC}_{50}$ or $\mathrm{LD}_{50}$ following 5-6 twofold dilutions $[39,56,57]$. Using these master stocks, the following volume-volume $(\mathrm{v} / \mathrm{v})$ mixtures of "Drug $\mathrm{A}$ " and "Drug B" were prepared: 0:4, 1:3, 1:1, 3:1, and 4:0. These mixtures were then two-fold serially diluted to generate a range of 8 concentrations in each case. Standard cytostatic or cytocidal antiplasmodial assay conditions (see above) were then followed to provide a dose response curve, and $\mathrm{IC}_{50}$ or $\mathrm{LD}_{50}$ for Drug A and Drug B in each v/v mixture was calculated [33,39,55-61]. Fractional inhibitory concentrations (FICs) were then calculated using Equations 1, 2 and 3 [39,55-57].

$$
\begin{aligned}
& \mathrm{FIC}_{\mathrm{A}}=\frac{\mathrm{IC}_{50} \text { of Drug A in Combination }}{\mathrm{IC}_{50} \text { of Drug A Alone }} \\
& \mathrm{FIC}_{\mathrm{B}}=\frac{\mathrm{IC}_{50} \text { of Drug B in Combination }}{\mathrm{IC}_{50} \text { of Drug B Alone }} \\
& \mathrm{FIC}_{\text {index }}=\mathrm{FIC}_{\mathrm{A}}+\mathrm{FIC}_{\mathrm{B}}
\end{aligned}
$$

Isobologram curves were constructed by plotting $\mathrm{FIC}_{\mathrm{B}}$ vs $\mathrm{FIC}_{\mathrm{A}}$. A straight diagonal line $\left(\mathrm{FIC}_{\text {index }}=1\right)$ indicates an additive effect between Drug A and Drug B [39,55-57], concave below the diagonal $\left(\mathrm{FIC}_{\text {index }}<1\right)$ indicates a synergistic effect and a convex curve above the diagonal $\left(\mathrm{FIC}_{\text {index }}>1\right)$ indicates antagonism.

\section{Results}

Similar to previous analyses of CQ, QN, QD, MQ and series of CQ and QN analogues [19,20,52], significant differences in cytostatic $\left(\mathrm{IC}_{50}\right)$ and cytocidal $\left(\mathrm{LD}_{50}\right)$ potency are observed for test compounds used in this work (Table 1, see also Additional files 1 and 2). Also similar to previous work [52], differences in drug sensitivity for CQS strain $\mathrm{HB} 3$ vs CQR strain Dd2 are not of similar magnitude when potency is defined via $\mathrm{IC}_{50} v s \mathrm{LD}_{50}$. For example, $\mathrm{Dd} 2$ is 2.4 -fold resistant to $\mathrm{PQ}$ via $\mathrm{IC}_{50}$ but approximately three-fold more sensitive via $\mathrm{LD}_{50}$ (Table 1 ). Dd2 shows similar cytostatic sensitivity to TQ compared to HB3, but interestingly is nearly four-fold more sensitive to cytocidal effects of TQ (Table 1). As reported previ-

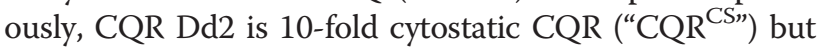

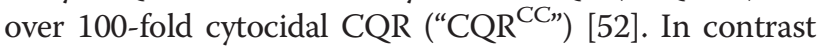
the related 4-aminoquinoline AQ shows similar potency against both strains, under either cytostatic or cytocidal assay conditions, which is quite rare behaviour for a quinoline anti-malarial drug based on previous examination of many quinoline compounds $[19,20,52]$. Similar to AQ, $\mathrm{MB}$ is quite active relative to quinolines under both cytostatic and cytocidal conditions and shows no major differences between HB3 and Dd2, making it a promising lead compound vs CQR P. falciparum.

Results of $\mathrm{IC}_{50^{-}}$and selected $\mathrm{LD}_{50^{-}}$-based isobologram analyses of these drugs in various combinations are shown in Figures 2 and 3 (see also Additional files 1, 2 and 3), with antagonism, additivity, and synergy findings summarized in Table 2. In contrast to Bray et al. [33] but in agreement with Akoachere et al. [39], the combination of CQ-PQ is antagonistic against HB3 (CQS) (black curve, Figure 2 top left) and additive against Dd2 (CQR) when potency is defined by $\mathrm{IC}_{50}$ (black curve, Figure 2 top right). This combination is strongly antagonistic against both strains when potency is defined by $\mathrm{LD}_{50}$ (Figure 3, black curves). $\mathrm{IC}_{50}$ antagonism is observed with the CQ-TQ combination against both strains (red curves, Figure 2 top) in contrast to synergy seen against the K1 strain (SE Asia, CQR) [33]. AQ combined with PQ is antagonistic against both strains by $\mathrm{IC}_{50}$. AQ-TQ is additive against HB3, but antagonistic against Dd2 (orange curves, middle panels Figure 2). CQ-AQ is cytostatically additive for both strains (Figure 2), but exhibits significant antag-

\begin{tabular}{|c|c|c|c|c|c|c|}
\hline \multirow[b]{2}{*}{ Drug } & \multicolumn{3}{|c|}{ Experimental I $C_{50}^{\mathrm{a}}(\mathrm{nM})$} & \multicolumn{3}{|c|}{ Experimental $\mathrm{LD}_{50}^{\mathrm{a}}(\mathrm{nM})$} \\
\hline & HB3 & $\mathrm{Dd} 2$ & $\mathbf{R}_{\mathrm{f}}^{\mathrm{b}}$ & HB3 & $\mathrm{Dd} 2$ & $\mathbf{R}_{\mathrm{f}}^{\mathbf{b}}$ \\
\hline $\mathrm{CQ}$ & $23.8(0.7)$ & $212.3(9.0)$ & 9.0 & $120.0(10.0)$ & $15300.0(900.0)$ & 127.5 \\
\hline $\mathrm{AQ}$ & $10.1(0.6)$ & $26.5(0.4)$ & 2.6 & $37.2(1.2)$ & $52.4(2.3)$ & 1.4 \\
\hline$P Q$ & $1990.0(16.6)$ & $4695.0(62.1)$ & 2.4 & $8640.0(50.0)$ & $2810.0(10.0)$ & 0.3 \\
\hline TQ & $2189.9(18.7)$ & $2092.2(20.0)$ & 1.0 & $42700.0(2042.6)$ & $12100.0(706.0)$ & 0.3 \\
\hline MB & $5.3(0.09)$ & $5.5(0.07)$ & 1.0 & $120.2(9.5)$ & $108.0(6.6)$ & 0.9 \\
\hline
\end{tabular}
onism against Dd2 under cytocidal conditions (Figure 3).

Table 1 Anti-plasmodial activity data

${ }^{a}$ Average of 3 independent measurements, each performed in triplicate (9 determinations total), with S.E.M. reported in parentheses.

${ }^{b}$ Resistance factor $\left(R_{f}\right)=D d 2$ value/HB3 value. 

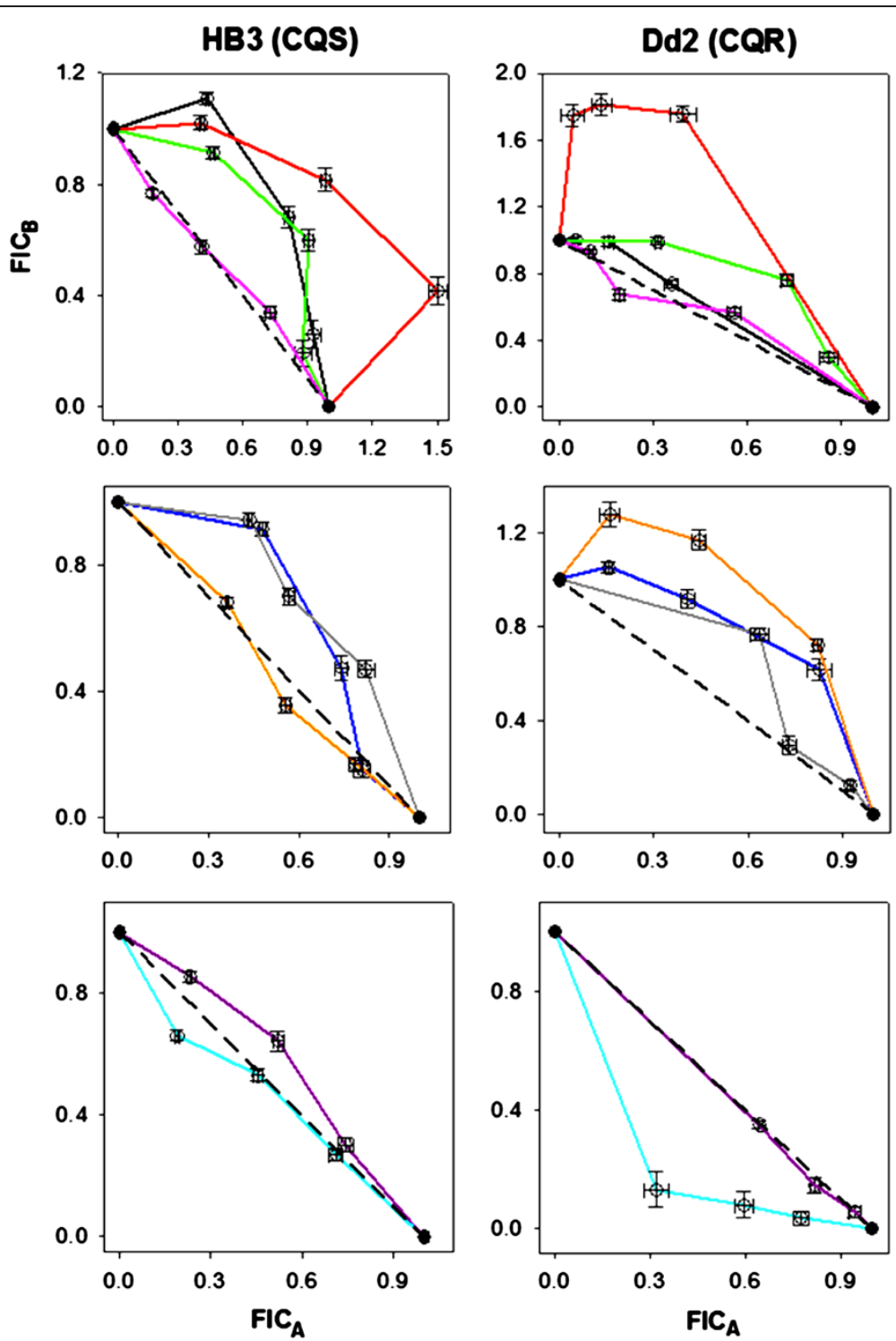

Figure $2 \mathrm{IC}_{50}$-based isobologram curves against HB3 (CQS, left column) and Dd2 (CQR, right column) for combinations with CQ (top), $\mathrm{AQ}$ (middle), and PQ/TQ (bottom) as "drug A" (x-axes). CQ-PQ (black), CQ-TQ (red), CQ-MB (green), CQ-AQ (pink), AQ-PQ (dark blue), AQ-TQ (orange), AQ-MB (gray), PQ-MB (purple), and TQ-MB (light blue or cyan). $\mathrm{FIC}_{\mathrm{A}}$ and $\mathrm{FIC}_{\mathrm{B}}$ correspond to the fractional inhibitory concentrations (see Methods) of the first and second drugs in each pair listed above, respectively. Error bars represent the standard error of the mean (S.E.M.) for duplicate experiments, each performed in triplicate (6 determinations total).

Overall, the important point is that synergy, antagonism, and additivity seen under cytostatic assay conditions are not necessarily conserved under cytocidal assay conditions. Similar to related conclusions based on multidrug resistance patterns [52] or drug accumulation studies [62], these data further highlight that the mechanism(s) of antimalarial drug parasite growth inhibition and parasite cell kill are not necessarily the same, and that molecular targets for the cytostatic vs cytocidal activities of some important anti-malarial drugs likely differ.

The $\mathrm{IC}_{50}$ antagonism observed for $\mathrm{CQ}-\mathrm{AQ}, \mathrm{CQ}-\mathrm{MB}$ and $\mathrm{AQ}-\mathrm{MB}$ combinations is in agreement with previous studies [33,40], except that in the case of AQ-MB, Garavito et al. previously measured additive effects for these two drugs against the FcM29 strain (Cameroon, CQR) [40]. IC 50 antagonism is also observed with PQ$\mathrm{MB}$ against $\mathrm{HB} 3$, but additivity is seen against $\mathrm{Dd} 2$, in agreement with Garavito et al. [40]. Finally, with the exception of $\mathrm{IC}_{50}$ additivity against HB3 (Figure 2 bottom left), excellent synergy is seen between $\mathrm{TQ}$ and $\mathrm{MB}$ under both cytostatic and cytocidal conditions, with synergy being significantly greater in the latter case (Figure 2). Importantly then, some drug combinations may show potent cytostatic and cytocidal synergy for 

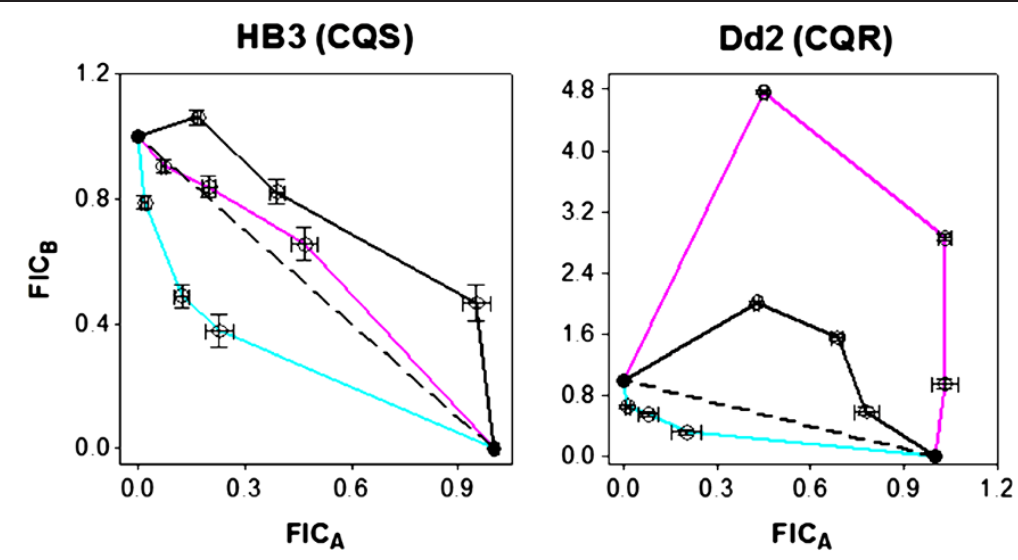

Figure 3 LD $_{50}$-based isobologram curves for HB3 (CQS, left) and Dd2 (CQR, right). CQ-PQ (black), CQ-AQ (pink), and TQ-MB (Cyan). FICA and $\mathrm{FIC}_{B}$ correspond to the fractional inhibitory concentrations (see Methods) of the first and second drugs in each pair listed above, respectively. Error bars represent the standard error of the mean (S.E.M.) for duplicate experiments, each performed in triplicate (6 determinations total).

CQR, but not CQS P. falciparum. This is an important concept for regions where CQR $P$. falciparum is dominant.

To further explore one contrasting result between these data and previous [33], the CQ-PQ and CQ-TQ combinations were reexamined against the FCB1 strain. This CQR strain from Thailand/S. Africa harbours the same $P$. falciparum chloroquine resistance transporter (PfCRT) amino acid mutations as $\mathrm{K} 1$ and has a similar CQ $\mathrm{IC}_{50}$ [63] (see Additional files 4 and 5). Dd2, however, differs from these two strains in a mutation at position 356, which is $\mathrm{I}$ in $\mathrm{K} 1$ and FCB1 but $\mathrm{T}$ in Dd2. Mutations in the $P$. falciparum multi-drug resistance protein (PfMDR1) are identical among these three strains [64,65] (see Additional file 6). Results obtained with FCB1 are similar to those obtained with HB3 and $\mathrm{Dd} 2$, in that combination of CQ with either PQ or TQ is antagonistic (Table 2, see also Additional file 5).

Table 2 Summary of drug interactions ${ }^{a}$

\begin{tabular}{|c|c|c|c|c|}
\hline \multirow[b]{2}{*}{ Combination } & \multicolumn{2}{|l|}{$I C_{50}$-Based } & \multicolumn{2}{|l|}{ LD $_{50}$-Based } \\
\hline & HB3 (CQS) & Dd2 (CQR) & HB3 (CQS) & Dd2 (CQR) \\
\hline $\mathrm{CQ}-\mathrm{PQ}$ & Antagonistic & Additive & Antagonistic & Antagonistic \\
\hline CQ-TQ & Antagonistic & Antagonistic & - & - \\
\hline CQ-AQ & Additive & Additive & Additive & Antagonistic \\
\hline CQ-MB & Antagonistic & Antagonistic & - & - \\
\hline$A Q-P Q$ & Antagonistic & Antagonistic & - & - \\
\hline AQ-TQ & Additive & Antagonistic & - & - \\
\hline AQ-MB & Antagonistic & Antagonistic & - & - \\
\hline PQ-MB & Antagonistic & Additive & - & - \\
\hline TQ-MB & Additive & Synergistic & Synergistic & Synergistic \\
\hline
\end{tabular}

${ }^{a}$ Antagonism is defined by a convex isobologram curve $\left(\mathrm{FIC}_{\text {index }}>1\right)$, additivity by a isobologram curve along the diagonal $\left(\mathrm{FIC}_{\text {index }}=1\right)$, and synergy by a concave isobologram curve $\left(\mathrm{FIC}_{\text {index }}<1\right)$ (Figure 2$)$.

\section{Discussion}

This study reports the first systematic analysis of quinoline anti-malarial drug combinations quantified for both cytostatic and cytocidal potency. Recent studies suggest that cytostatic and cytocidal mechanisms of action for some anti-malarial drugs differ [19,20,52,62], and the present data further support this concept. Comparative investigation of drug combinations under both $\mathrm{IC}_{50}$ and $\mathrm{LD}_{50}$ conditions will enhance further development of effective combination therapies and also increase understanding of antimalarial drug pharmacology and resistance. $\mathrm{IC}_{50}$ and $\mathrm{LD}_{50}$ data for the individual drugs reinforces this concept (Table 1). Strain Dd2 is 10 -fold resistant to CQ (relative to strain $\mathrm{HB} 3$ ) when $\mathrm{IC}_{50}$ is ratio'd, but over 100 -fold resistant when $\mathrm{LD}_{50}$ values are ratio'd. On the other hand, the closely-related 4-aminoquinoline AQ has similar potency for both strains under both cytostatic and cytocidal conditions, a characteristic also found for ART (A. Gorka, P. Roepe, unpublished). MB, while having nearly identical activity for the CQS and CQR strains (behavior similar to AQ), is about 20-fold less potent by $\mathrm{LD}_{50}$ relative to $\mathrm{IC}_{50}$ (unlike $\mathrm{AQ}$ ). $\mathrm{PQ}$, an 8aminoquinoline known to be a poor blood schizonticide $[17,18]$, is nonetheless active compared to CQ when potency is defined by $\mathrm{LD}_{50}$ for CQR strain $\mathrm{Dd} 2(15.3 \mu \mathrm{M}$ CQ $\mathrm{LD}_{50}$ vs $2.8 \mu \mathrm{M}$ PQ $\mathrm{LD}_{50}$, Table 1). In fact, $\mathrm{Dd} 2$ is about three-fold hypersensitive to $\mathrm{PQ}$ relative to $\mathrm{HB} 3$ when $\mathrm{LD}_{50}$ are compared. Results for drug combinations show significant differences in synergy, additivity, and antagonism (Table 2), as well as in the shapes of their isobologram curves (Figure 2). In general, cytocidal antagonism is significantly more pronounced relative to examples of cytostatic antagonism. Overall, these data offer additional explanations for why in vitro $\mathrm{IC}_{50}$-based assessment of mono- or combination therapy may not always 
predict results in the clinic, where both cytostatic and cytocidal potencies are critical.

The CQ-PQ combination results in cytostatic antagonism (CQS) and additivity (CQR), which disagrees with data [33] for the CQR strain K1, where potent synergy for CQ-PQ and CQ-TQ combinations was found. In growth inhibition assays, as suggested [33], the combination effects may be PfCRT-dependent and rely on the presence of the $\mathrm{PQ} / \mathrm{TQ}$ basic side chain, as well as coadministration of the drug pair [33]. As suggested [33], due to their structural similarity to CQ but increased lipophilicity, PQ and TQ may bind with higher affinity to the mutated pore of PfCRT and block passage of CQ [33]. However, compared to strain Dd2, the K1 strain (CQR, S.E. Asia) differs in a PfCRT mutation at position 356 (see Additional file 4) [63]. Although strain K1 was not available for this study, CQ-PQ and CQ-TQ combinations were re-tested against the FCB1 strain (CQR, Thailand/S. Africa), which bears an identical set of PfCRT [63] and PfMDR1 [64,65] mutations relative to K1. These combinations again exhibited antagonism (see Additional file 5), indicating altered CQ transport by PfCRT may not be the only factor governing combination effects.

The closely-related 4-aminoquinoline AQ also showed antagonism with $\mathrm{PQ}$ and TQ across all conditions, with the exception of AQ-TQ against Dd2, which was additive. Therefore, similar to the CQ combinations discussed above, differences between cytostatic and cytocidal response, as well as important differences between the CQS and CQR strains are observed when $A Q$ is a partner drug. Further exploration of these concepts with other CQS and CQR strains is vital. Combination of CQ with AQ was purely additive against both strains by $\mathrm{IC}_{50}$ and against the CQS strain by $\mathrm{LD}_{50}$ (Figures 2 and 3, Table 2). AQ is expected to have a similar overall mechanism of action to that of CQ under cytostatic conditions (e.g. inhibition of $\mathrm{Hz}$ formation), so additive action of these two drugs in combination is expected for $\mathrm{IC}_{50}$. Surprisingly though, CQ-AQ exhibits significant antagonism against the $\mathrm{CQR} D \mathrm{D} 2$ strain by $\mathrm{LD}_{50}$. In light of recent evidence $[19,20,52,62]$, this observation again points to the general hypothesis that drug targets and the manner in which the parasite facilitates resistance to those drugs are different under cytocidal $v s$ cytostatic conditions. In investigating this further, future work will test for synergy, additivity, and antagonism for these drug pairs against a wider range of strains harbouring different CQR-associated PfCRT alleles.

CQ-MB was cytostatically antagonistic against both strains, in agreement with Akoachere et al., who observed antagonism against 3D7 (CQS) and K1 [39], but in contrast to Garavito et al., who observed additivity against the FcM29 strain (CQR, Cameroon) [40]. Antagonism between $C Q$ and $M B$ is surprising given the high blood schizonticidal potency of $\mathrm{MB}$, its potent inhibition of $\mathrm{Hz}$ formation in vitro [41,42], and inhibition of parasite glutathione reductase $[46-48,66,67]$, as well as its ability to accumulate in the DV, similar to CQ [42]. However, such antagonism has also been reported in the field, with failure of CQ and MB observed in treatment of uncomplicated malaria in young children in Burkina Faso $[49,50]$. AQ was also antagonistic with $\mathrm{MB}$, in agreement with Garavito et al. [40]. $\mathrm{IC}_{50}$ antagonism in such cases might indicate distinctly different modes of haemozoin precursor binding [68].

\section{Conclusions}

Using a rigorous isobologram approach, cytocidal and cytostatic anti-plasmodial potency have been determined for various combinations of quinoline drugs and $\mathrm{MB}$, for both a CQS and CQR strain of P. falciparum. The results highlight differences in drug activity and resistance under cytostatic vs cytocidal conditions. They also show that antagonism or synergy found for a drug pair via $\mathrm{IC}_{50}$ data (cytostatic activity) does not necessarily predict the same behavior when potency for the pair is quantified via $\mathrm{LD}_{50}$ data (cytocidal activity). Furthermore, the data help prioritize combinations that warrant further exploration against CQR malaria (e.g. TQ-MB), vs those with limited applicability (e.g. CQ-PQ). Important next steps are to examine the correlation between anti-malarial drug combination effects and $\mathrm{Hz}$ inhibition, as well as to widen this cytostatic $v s$ cytocidal profiling to other drugs, active drug metabolites, and many geographic isolates of P. falciparum.

\section{Additional files}

Additional file 1: $I C_{50}$ data for all drug combinations tested against HB3 and Dd2.

Additional file 2: $\mathrm{LD}_{50}$ data for all drug combinations tested against $\mathrm{HB} 3$ and Dd2.

Additional file 3: Results of $\mathrm{IC}_{50}$-based drug combination analyses. All FIC data is shown with relevant statistics.

Additional file 4: PfCRT amino acid substitutions associated with CQS and CQR PfCRT isoforms in Plsmodium falciparum.

Additional file 5: $I C_{50}$ data for all drug combinations tested against FCB1.

Additional file 6: PfMDR1 amino acid substitutions associated with CQS and CQR PFMDR1 isoforms in Plasmodium falciparum.

\section{Abbreviations}

ACT: Artemisinin combination therapy; AQ: Amodiaquine; ART: Artemisinin; $\mathrm{CQ}$ : Chloroquine; $\mathrm{CQR}$ : Chloroquine resistant; $\mathrm{CQR}^{\mathrm{CC}}$ : Cytocidal chloroquine resistance; CQS: Chloroquine sensitive; $\mathrm{CQS}^{\mathrm{CC}}$ : Cytostatic chloroquine resistance; DV: Digestive vacuole; FIC: Fractional inhibitory concentration; G6PD: Glucose-6-phosphate dehydrogenase; GR: Glutathione reductase; Hz: Haemozoin; MB: Methylene blue; MQ: Mefloquine; QN: Quinine; PfCRT: Plasmodium falciparum chloroquine resistance transporter; 
PfMDR1: Plasmodium falciparum multi-drug-resistance protein;

PQ: Primaquine; TQ: Tafenoquine; vs: Versus.

\section{Competing interests}

The authors declare they have no competing interests.

\section{Authors' contributions}

APG carried out antiplasmodial and isobologram assays and data analysis, assisted in interpretation of the results, and drafted the manuscript. LM maintained $P$. falciparum cultures, participated in anti-plasmodial and isobologram assays and data analysis, and assisted manuscript preparation. PDR conceived of the study, interpreted results, and drafted the manuscript. All authors have read and approved the final manuscript.

\section{Acknowledgements}

We gratefully acknowledge Ms. Katy Sherlach for helpful discussions and literature assistance. We also acknowledge NIH grant AlO56312 for financial support.

\section{Author details}

${ }^{1}$ Department of Chemistry, Department of Biochemistry, Cellular, \& Molecular Biology, and Center for Infectious Disease, Georgetown University, 37th and 'O' Sts. NW, Washington, DC 20057, USA. ²Present address: Chemical Biology Laboratory, National Cancer Institute, 376 Boyles Street, Frederick, MD 21702 USA.

Received: 14 June 2013 Accepted: 18 August 2013

Published: 18 September 2013

\section{References}

1. World Health Organization: World malaria report. Geneva,Switzerland: World Health Organization; 2010. http://www.who.int/malaria/publications/atoz/ 9789241564106/en/index.html.

2. Roepe PD: Molecular and physiologic basis of quinoline drug resistance in Plasmodium falciparum malaria. Future Microbiol 2009, 4:441-455.

3. Cheeseman $I_{\text {, }}$ Miller BA, Nair S, Nkhoma S, Tan A, Tan JC, Al Saai S, Phyo $A P, M o o ~ C L$, Lwin KM, McGready R, Ashley E, Imwong M, Stepniewska K, Yi P, Dondorp AM, Mayxay M, Newton PN, White NJ, Nosten F, Ferdig MT, Anderson TJ: A major genome region underlying artemisinin resistance in malaria. Science 2012, 336:79-82.

4. Wells TN, Alonso PL, Gutteridge WE: New medicines to improve control and contribute to the eradication of malaria. Nat Rev Drug Discov 2009, 8:879-891.

5. WHO: Guidelines for the treatment of malaria. 2nd edition. Geneva, Switzerland: World Health Organization; 2010. http://www.who.int/malaria/ publications/atoz/9789241547925/en/index.html.

6. White NJ: Primaquine to prevent transmission of falciparum malaria. Lancet Infect Dis 2009, 13:175-181.

7. Dechy-Cabaret O, Benoit-Vical F: Effects of antimalarial molecules on the gametocyte stage of Plasmodium falciparum: the debate. J Med Chem 2012, 55:10328-10344

8. Pukrittayakamee S, Vanijanonta S, Chantra A, Clemens R, White NJ: Blood stage antimalarial efficacy of primaquine in Plasmodium vivax malaria. J Infect Dis 1994, 169:932-935.

9. Krotoski WA, Garnham PC, Cogswell FB, Collins WE, Bray RS, Gwasz RW, Killick-Kendrick R, Wolf RH, Sinden R, Hollingdale M: Observations on early and late post-sporozoite tissue stages in primate malaria. IV. Preerythrocytic schizonts and/or hypnozoites of Chesson and North Korean strains of Plasmodium vivax in the chimpanzee. Am J Trop Med Hyg 1986, 35:263-274

10. Wells TNC, Burrows JN, Baird JK: Targeting the hypnozoite reservoir of Plasmodium vivax: the hidden obstacle to malaria elimination. Trends Parasitol 2010, 26:145-151.

11. Arnold J, Alving AS, Hockwald RS, Clayman CB, Dern RJ, Beutler E, Flanagan $\mathrm{CL}$, Jeffery $\mathrm{GM}$ : The antimalarial action of primaquine against the blood and tissue stages of falciparum malaria (Panama, P-F-6 strain). J Lab Clin Med 1955, 46:391-397.

12. Peatey CL, Skinner-Adams TS, Dixon MWA, McCarthy JS, Gardiner DL, Trenholme KR: Effect of antimalarial drugs on Plasmodium falciparum gametocytes. J Infect Dis 2009, 200:1518-1521.
13. Peatey $C L$, Spicer TP, Hodder PS, Trenholme KR, Gardiner DL: A highthroughput assay for the identification of drugs against late-stage Plasmodium falciparum gametocytes. Mol Biochem Parasitol 2011, 180:127-131.

14. Rieckmann KH, McNamara JV, Frischer H, Stockert TA, Carason PE, Powel RD: Gametocytocidal and sporontocidal effects of primaquine and of sulfadiazine with pyrimethamine in a chloroquine-resistant strain of Plasmodium falciparum. Bull World Health Organ 1968, 38:625-632.

15. Chotivanich K, Sattabongkot J, Udomsangpetch R, Looareesuwan S, Day NPJ, Coleman RE, White NJ: Transmission-blocking activities of quinine, primaquine, and artesunate. Antimicrob Agents Chemother 2006, 60:1927-1930.

16. Eziefula AC, Gosling R, Hwang J, Hsiang MS, Bousema T, Seidlein L, Drakely C: Rationale for short course primaquine in Africa to interrupt malaria transmission. Malar J 2011, 11:360.

17. Basco LK, Bickii J, Ringwald P: In-vitro activity of primaquine against the asexual blood stages of Plasmodium falciparum. Ann Trop Med Parasitol 1999, 93:179-182.

18. Kaur K, Jain M, Khan SI, Jacob MR, Tekwani BL, Singh S, Singh PP, Jain R: Synthesis, antiprotozoal, antimicrobial, $\beta$-hematin inhibition, cytotoxicity and methemoglobin (metHb) formation activities of bis(8aminoquinolines). Bioorg Med Chem 2011, 19:197-210.

19. Gorka AP, Alumasa JN, Sherlach KS, Jacobs LM, Nickley KB, Brower JP, de Dios AC, Roepe PD: Cytostatic versus cytocidal activities of chloroquine analogues and inhibition of hemozoin crystal growth. Antimicrob Agents Chemother 2013, 57:356-364.

20. Gorka AP, Sherlach KS, de Dios AC, Roepe PD: Relative to quinine and quinidine, their 9-epimers exhibit decreased cytostatic activity and altered heme binding but similar cytocidal activity versus Plasmodium falciparum. Antimicrob Agents Chemother 2013, 57:365-374.

21. Dorn A, Vippagunta SR, Matile H, Jaquet C, Vennerstrom JL, Ridley RG: An assessment of drug-haematin binding as a mechanism for inhibition of haematin polymerisation by quinoline antimalarials. Biochem Pharmacol 1998, 55:727-736.

22. Egan TJ, Ross DC, Adams PA: Quinoline antimalarial drugs inhibit spontaneous formation of $\beta$-hematin (malaria pigment). FEBS Lett 1994, 352:54-57.

23. Parapini S, Basilico N, Pasini E, Egan TJ, Olliaro P, Taramelli D, Monti D: Standardization of the physicochemical parameters to assess in vitro the $\beta$-hematin inhibitory activity of antimalarial drugs. Exp Parasitol 2000, 96:249-256.

24. Cappellini MD, Fiorelli G: Glucose-6-phosphate dehydrogenase deficiency. Lancet 2008, 371:64-67.

25. White NJ, Qiao LG, Qi G, Luzzatto L: Rationale for recommending a lower dose of primaquine as a Plasmodium falciparum gametocytocide in populations where G6PD deficiency is common. Malar J 2012, 11:418.

26. Ezifula AC, Gosling R, Hwang J, Hsiang MS, Bousema T, Seidlein L, Drakeley C: Rationale for short course primaquine in Africa to interrupt malaria transmission. Malar J 2012, 11:360.

27. Mihaly GW, Ward SA, Edwards G, Nicholl DD, L'E Orme M, Breckenridge AM: Pharmacokinetics of primaquine in man. I. Studies of the absolute bioavailability and effects of dose size. Br J Clin Pharmac 1985, 19:745-750.

28. Shanks GD, Oloo AJ, Aleman GM, Ohrt C, Klotz FW, Braitman D, Horton J, Brueckner R: A new primaquine analogue, tafenoquine (WR 238605), for prophylaxis against Plasmodium falciparum malaria. Clin Infect Dis 2001, 33:1968-1974

29. Pradines B, Mamfoumbi MM, Tall A, Sokhna C, Koeck JL, Fusai T, Mosnier J, Czarnecki E, Spiegel A, Trape JF, Kombila M, Rogier C: In vitro activity of tafenoquine against the asexual blood stages of Plasmodium falciparum isolates from Gabon, Senegal, and Djibouti. Antimicrob Agents Chemother 2006, 50:3225-3226

30. Brueckner RP, Lasseter KC, Lin ET, Schuster BG: First-time-in-humans safety and pharmacokinetics of WR 238605, a new antimalarial. Am J Trop Med Hyg 1998, 58:645-649.

31. Edstein MD, Kocisko DA Walsh DS, Eamsila C, Charles BG, Rieckmann KH: Plasma concentrations of tafenoquine, a new long-acting antimalarial agent, in Thai soldiers receiving monthly prophylaxis. Clin Infect Dis 2003, 37:1654-1658

32. Myint HY, Berman J, Walker L, Pybus B, Melendez V, Baird JK, Ohrt C: Improving the therapeutic index of 8-aminoquinolines by the use of drug combinations: review of the literature and proposal for future investigations. Am J Trop Med Hyg 2011, 85:1010-1014. 
33. Bray PG, Deed S, Fox E, Kalkanidis M, Mungthin M, Deady LW, Tilley L: Primaquine synergizes the activity of chloroquine against chloroquineresistant $P$. falciparum. Biochem Pharmacol 2005, 70:1158-1166.

34. Baird JK, Wiady I, Sutanihardja A, Suradi D, Suradi P, Basri H, Sekartuti, Ayomi E, Fryauff DJ, Hoffman SL: Therapeutic efficacy of chloroquine combined with primaquine against Plasmodium falciparum in Northeastern Papua, Indonesia. Am J Trop Med Hyg 2002, 66:659-660.

35. Kawai A, Arita N, Matsumoto Y, Kawabata M, Chowdhury MS, Saito-Ito A: Efficacy of chloroquine plus primaquine treatment and pfcrt mutation in uncomplicated falciparum malaria patients in Rangamati, Bangladesh. Parasitol Int 2011, 60:341-346.

36. Baird JK, Basri H, Subianto B, Fryauff DJ, McElroy PD, Leksana B, Richie TL, Masbar S, Wignall FS, Hoffman SL: Treatment of chloroquine-resistant Plasmodium vivax with chloroquine and primaquine or halofantrine. J Infect Dis 1995, 171:1678-1682.

37. Ehrlich P, Guttmann P: Ueber die Wirkung des Methylenblau bei Malaria. Berliner klinische Wochhenschrift 1891, 28:953-956.

38. Adjalley SH, Johnston GL, Li T, Eastman RT, Ekland EH, Eappen AG, Richman A, $\operatorname{Sim}$ B, KL L, MCS H, SL F, DA: Quantitative assessment of Plasmodium falciparum sexual development reveals potent transmission-blocking activity by methylene blue. Proc Natl Acad Sci U S A 2011, 108:E1214-E1223.

39. Akoachere M, Buchholz K, Fischer E, Burhenne J, Haefeli WE, Schirmer RH, Becker K: In vitro assessment of methylene blue on chloroquine-sensitive and -resistant Plasmodium falciparum strains reveals synergistic action with artemisinins. Antimicrob Agents Chemother 2005, 49:4592-4597.

40. Garavito G, Bertani S, Rincon J, Maurel S, Monje MC, Landau I, Valentin A, Deharo E: Blood schizontocidal activity of methylene blue in combination with antimalarials against Plasmodium falciparum. Parasite 2007, 14:135-140.

41. Schirmer RH, Coulibaly B, Stich A, Scheiwein M, Merkle H, Eubel J, Becker K, Becher H, Muller O, Zich T, Schiek W, Kouyate B: Methylene blue as an antimalarial agent. Redox Rep 2003, 8:272-275

42. Atamna H, Krugliak M, Shalmiev G, Deharo E, Pescarmona G, Ginsburg H: Mode of antimalarial effect of methylene blue and some of its analogues on Plasmodium falciparum in culture and their inhibition of $P$. vinckei petteri and P. yoelii nigeriensis in vivo. Biochem Pharmacol 1996, 51:693-700

43. Vennerstrom JL, Makler MT, Angerhofer CK, Williams JA: Antimalarial dyes revisited: xanthenes, azines, oxazines, and thiazines. Antimicrob Agents Chemother 1995, 39:2671-2677.

44. Pastrana-Mena R, Dinglasan RR, Franke-Fayard B, Vega-Rodríguez J, FuentesCaraballo M, Baerga-Ortiz A, Coppens I, Jacobs-Lorena M, Janse CJ, Serrano AE: Glutathione reductase-null malaria parasites have normal blood stage growth but arrest during development in the mosquito. $J$ Biol Chem 2010, 285:27045-27056.

45. Antsey NM, Hassanali MY, Mlalasi J, Manyenga D, Mwaikambo ED: Elevated levels of methaemoglobin in Tanzanian children with severe and uncomplicated malaria. Trans R Soc Trop Med Hyg 1996, 90:147-151.

46. Farber PM, Arscott LD Jr, Williams $\mathrm{CH}$, Becker K, Schirmer RH: Recombinant Plasmodium falciparum glutathione reductase is inhibited by the antimalarial dye methylene blue. FEBS Lett 1998, 422:311-314.

47. Buchholz K, Schirmer RH, Eubell JK, Akoachere MB, Dandekar T, Becker K, Gromer S: Interactions of methylene blue with human disulfide reductases and their orthologues from Plasmodium falciparum. Antimicrob Agents Chemother 2008, 52:183-191.

48. Davioud-Charvet E, Delarue S, Biot C, Schwöbel B, Boehme CC, Müssigbrodt A, Maes L, Sergheraert C, Grellier P, Schirmer RH, Becker KA: Prodrug form of a Plasmodium falciparum glutathione reductase inhibitor conjugated with a 4-anilinoquinoline. J Med Chem 2001, 44:4268-4276.

49. Meissner PE, Mandi G, Coulibaly B, Witte S, Tapsoba T, Mansmann U, Rengelhausen J, Schiek W, Jahn A, Walter-Sack I, Mikus G, Burhenne J, Reidel K-D, Schirmer RH, Kouyate B, Muller O: Methylene blue for malaria in Africa: results from a dose-finding study in combination with chloroquine. Malar J 2006, 5:84

50. Coulibaly B, Zoungrana A, Mockenhaupt FP, Schirmer RH, Klose C, Mansmann U, Meissner PE, Muller O: Strong gametocytocidal effect of methylene blue-based combination therapy against falciparum malaria: a randomised controlled trial. PLoS One 2009, 4:e5318

51. Zoungrana A, Coulibaly B, Sié A, Walter-Sack I, Mockenhaupt FP, Kouyaté B, Schirmer RH, Klose C, Mansmann U, Meissner P, Müller O: Safety and efficacy of methylene blue combined with artesunate or amodiaquine for uncomplicated falciparum malaria: a randomized controlled trial from Burkina Faso. PLoS One 2008, 3:e1630.

52. Paguio MF, Bogle KL, Roepe PD: Plasmodium falciparum resistance to cytocidal versus cytostatic effects of chloroquine. Mol Biochem Parasitol 2011, 178:1-6.

53. Trager W, Jensen WB: Human malarial parasites in continuous culture. Science 1976, 193:673-675.

54. Bennett TN, Paguio M, Gligorijevic B, Seudieu C, Kosar AD, Davidson E, Roepe PD: Novel, rapid, and inexpensive cell-based quantification of antimalarial drug efficacy. Antimicrob Agents Chemother 2004, 48:1807-1810.

55. Fivelman QL, Adagu IS, Warhurst DC: Modified fixed-ratio isobologram method for studying in vitro interactions between atovaquone and proguanil or dihydroartemisinin against drug-resistant strains of Plasmodium falciparum. Antimicrob Agents Chemother 2004, 48:4097-4102.

56. Kelly JX, Smilkstein MJ, Cooper RA, Lane KD, Johnson RA, Janowsky A, Dodean RA, Hinrichs DJ, Winter R, Riscoe M: Design, synthesis, and evaluation of $10-\mathrm{N}$-substituted acridones as novel chemosensitizers in Plasmodium falciparum. Antimicrob Agents Chemother 2007, 51:4133-4140.

57. Kelly JX, Smilkstein MJ, Brun R, Wittlin S, Cooper RA, Lane KD, Janowsky A, Johnson RA, Dodean RA, Winter R, Hinrichs DJ, Riscoe MK: Discovery of dual function acridones as a new antimalarial chemotype. Nature 2009, 459:270-273.

58. Berenbaum MC: A method for testing for synergy with any number of agents. J Infec Dis 1978, 137:122-130.

59. Adovelande J, Deleze J, Schrevel J: Synergy between two calcium channel blockers, verapamil and fantofarone (SR33557), in reversing chloroquine resistance in Plasmodium falciparum. Biochem Pharmacol 1998, 55:433-440.

60. Wagenpfeil S, Trieber U, Lehmer A: Isobologram analysis in MATLAB for combined effects of two agents in dose-response experiments. In Medical Data Analysis: $4^{\text {th }}$ International Symposium, ISMDA 2003, Berlin, Germany, LNCS 2868, Volume 4. Edited by Perner P, Brause R, Holzhutter H-G. Berlin: Springer-Verlag Berlin Heidelberg; 2003:47-54

61. Tallarida RJ: An overview of drug combination analysis with isobolograms. J Pharmacol Exp Ther 2006, 319:1-7.

62. Cabrera M, Paguio MF, Xie C, Roepe PD: Reduced digestive vacuolar accumulation of chloroquine is not linked to resistance to chloroquine toxicity. Biochemistry 2009, 48:11152-11154.

63. Fidock DA, Nomura T, Talley AK, Cooper RA, Dzekunov SM, Ferdig MT, Ursos LM, Sidhu AB, Naudé B, Deitsch KW, Su XZ, Wootton JC, Roepe PD, Wellems TE: Mutations in the $P$. falciparum digestive vacuole transmembrane protein PfCRT and evidence for their role in chloroquine resistance. Mol Cell 2000, 6:861-871.

64. Foote SJ, Kyle DE, Martin RK, Oduola AM, Forsyth K, Kemp DJ, Cowman AF: Several alleles of the multidrug-resistance gene are closely linked to chloroquine resistance in Plasmodium falciparum. Nature 1990, 345:255-258.

65. Sisowath C, Petersen I, Veiga MI, Mårtensson A, Premji Z, Björkman A, Fidock DA, Gil JP: In vivo selection of Plasmodium falciparum parasites carrying the chloroquine-susceptible pfort $\mathrm{K} 76$ allele after treatment with artemether-lumefantrine in Africa. J Infect Dis 2009, 199:750-757.

66. Meirjohann S, Walter RD, Muller S: Regulation of intracellular glutathione levels in erythrocytes infected with chloroquine-sensitive and chloroquineresistant Plasmodium falciparum. Biochem J 2002, 368:761-768.

67. Ginsburg H, Golenser J: Glutathione is involved in the antimalarial action of chloroquine and its modulation affects drug sensitivity of human and murine species of Plasmodium. Redox Rep 2003, 8:276-279.

68. Gorka AP, de Dios A, Roepe PD: Quinoline drug-heme interactions and implications for antimalarial cytostatic versus cytocidal activities. J Med Chem 2013. April 29 [Epub ahead of print] PMID 23586757.

doi:10.1186/1475-2875-12-332

Cite this article as: Gorka et al:: Cytostatic versus cytocidal profiling of quinoline drug combinations via modified fixed-ratio isobologram analysis. Malaria Journal 2013 12:332. 